\title{
Point prevalence of complications between the $Y$ connection technique and the usual care technique for blood restitution in patients of an outpatient hemodialysis unit: a comparison
}

This article was published in the following Dove Press journal: International Journal of Nephrology and Renovascular Disease 21 June 2017

Number of times this article has been viewed

\author{
Nadine Tacchini-Jacquier ${ }^{\prime}$ \\ Henk Verloo ${ }^{2,3}$ \\ 'Development of Nursing Practices \\ Unit, Valais Hospital, ${ }^{2}$ School of Health \\ sciences, HES-SO Valais - Wallis, \\ University of Applied Sciences and \\ Arts Western Switzerland; ${ }^{3}$ Nursing \\ Department, Valais Hospital, Sion, \\ Switzerland
}

Background: Central venous catheter-related infections (CVCIs) in patients on maintenance hemodialysis (HD) have been documented due to unsafe/unsterile manipulations by nurses during HD machine deconnection and reconnection. Given the gravity of CVCIs in HD patients using catheter access, precise, safe installation of the device, and good nursing technique are crucial. Aim: To assess and compare safety performance and complications of a Y-connection $(n=133)$ versus the usual tunneled central venous catheter (CVC) technique $(n=73)$ among HD patients and then explore preferences between techniques among patients and frontline HD nurses.

Materials and methods: A prospective, comparative, 3-month point prevalence survey was conducted among HD outpatients and frontline HD nurses in a 600-bed teaching hospital in the canton of Valais.

Results: Nine HD outpatients (average age, 68.3 years; $\mathrm{SD}=12.3$ ) were recruited. The two techniques showed no differences in C-reactive protein levels $(p=0.465)$, pain $(p=1.00)$, or local complications due to dressings soiled by exudate at the catheter insert point $(p=0.066)$. The relative risk ratio $(\mathrm{RR})$ indicated that $\mathrm{CVCI}$ was 1.667 times $(95 \% \mathrm{CI} ; 0.437,6.358, p=0.50)$ more likely with a Y-connection. Neither the Y-connection technique (RR 1.63; [95\% CI; 0.554, 4.790]; $p=0.32$ ) nor usual CVC technique (RR 0.58; [95\% CI; 0.277, 1.217]; $p=0.13$ ) were significant relative risk factors for complications. Fifty-seven percent of HD patients stated that they felt more secure and comfortable using the Y-connection technique than the usual care technique. Eleven of the 12 nurses involved preferred the Y-connection technique, feeling that is was safer and easier in use.

Conclusion: No difference was found in the complication rates of two blood restitution techniques - the Y-connection versus the usual CVC technique. HD outpatients and nurses preferred the Y-connection for blood restitution.

Keywords: patient preferences, clinical expertise, complications among HD patients, satisfaction evidence-based practice, nursing, central venous catheter, nursing technics

\section{Introduction/background}

A recent epidemiological survey indicated that the canton of Valais had an elevated prevalence of chronic dialysis patients compared to the national mean, with 476 per million inhabitants, compared to 329 in Switzerland overall. ${ }^{1-3}$ The mean age at the start of therapy was 61 years old $(\mathrm{SD}=14) .{ }^{1}$ Among the different etiologies, hereditary diseases were more frequently documented than vascular nephropathies. ${ }^{2}$ Valais had fewer patients on peritoneal dialysis ( $6 \%$ vs $19 \%$ ) and transplant waiting lists (12\% vs
Correspondence: Nadine

Tacchini-Jacquier

Development of Nursing Practices Unit, Valais Hospital, Avenue du Grand-

Champsec, $86 \mathrm{CH}-195 \mathrm{I}$ Sion, Switzerland

Tel +4l 276036752

Email nadine.tacchini@hopitalvs.ch 
$16 \%$ ) than Switzerland's statistical average. ${ }^{3}$ Approximately $91.9 \%$ of patients diagnosed with end-stage renal disease (ESRD) received maintenance hemodialysis (HD) treatment as renal replacement therapy. ${ }^{3,4}$ This intervention was typically prescribed three times per week, for $4-6 \mathrm{~h}$ per session, and continued throughout the patient's lifetime or until a successful kidney transplantation. ${ }^{1}$ Although advances in HD treatment have extended the lifespan of ESRD patients, this treatment alone cannot ensure the preservation of quality of life (QOL). ${ }^{2} \mathrm{HD}$ patients have typically suffered from significant impairments to QOL (with important risks of morbidity and mortality due to the infection of vascular access points) when compared to their healthy counterparts or those who have undergone successful kidney transplants., ${ }^{2,5}$

Vascular access is not only known as the obvious Achilles' heel of HD patients but it is also considered as a determinant of patient outcomes. ${ }^{6,7}$ The prevalence of central venous catheter (CVC) use has been documented at $>80 \%$ among chronic HD patients. ${ }^{8}$ There is a substantially increased risk of chronic HD patients suffering from catheter-associated infections and dysfunction, and the literature documents high morbidity and mortality rates. ${ }^{9}{ }^{10}$ In addition, hospital admissions due to vascular-access infections have more than doubled in recent decades. ${ }^{11}$ Obviously, CVC-related infections (CVCIs) and adverse complications have been associated with high rates of hospitalization, high treatment costs, and poor survival. ${ }^{8}$ The incidence of CVCIs ranges between 0.6 and 6.5 episodes per 1,000 catheter days. ${ }^{12}$ Compared with patients with an arteriovenous fistula, those with a tunneled CVC have a 15 times greater risk of $\mathrm{CVCI}$ and an all-cause mortality rate ranging from $12 \%$ to $25 \% .^{10} \mathrm{CVCIs}$ encompass exit-site infections, tunnel infections, and bacteremia due to unsafe/unsterile manipulations by nurses during HD deconnection and reconnection. ${ }^{13}$ The two main routes by which organisms get into the bloodstream and cause CVCI are the extraluminal and intraluminal pathways. ${ }^{14}$ The extraluminal pathway involves contact between skin-surface organisms and the external surface of the catheter at the time of the insertion or afterward, but before complete exit-site healing of the subcutaneous tunnel. ${ }^{9}$

Identified risk factors for CVCI include poor-patient hygiene, previous CVCI, recent hospitalization, long-term catheter use, inadequate dialysis, hypoalbuminemia, nasal carriage of Staphylococcus aureus, diabetes mellitus, an immunocompromised status, atherosclerosis, and hypertension. Serious metastatic infectious complications occur in $3 \%-44 \%$ of episodes and can include endocarditis, osteomyelitis, thrombophlebitis, septic arthritis, spinal epidural abscess, and large atrial thrombi. ${ }^{5,15}$ Given the gravity of
CVCIs in HD patients using catheter access, applying precise, safe nursing techniques seems crucial. ${ }^{13}$ However, this seemingly basic requirement has been challenged by the variety of newly developed connection systems between the CVC and the HD machine. The literature describes the use of symptoms, signs, and biological parameters with which to objectively measure the determinants guiding the performance of CVC connection techniques. However, little research has explored nurses' opinions of the ease of use of different CVC connections as a factor to decrease the risk of CVCI or their feelings about safety and the preferences of HD patients. ${ }^{16}$ The HD unit nursing staff in the present study wished to implement evidence-based practice (EBP) with their patients, integrating scientific knowledge, nurses' clinical expertise, and patient preferences. ${ }^{17}$ Bearing in mind that ICU nurses prefer an Y-connection compared to the standard connection for HD among acute renal failure for all age ICU-patients in the Valais Hospitals, standard connection technique is been challenged by the HD team members on the security and adverse complications among chronic HD patients. With this in mind, they expressed an urgent need to clinically assess the pertinence and safety of the standard techniques and connection materials. ${ }^{18}$

As part of an EBP strategy, the present study aimed to assess and compare the safety performance and other potential complications of a Y-connection and the usual tunneled CVC connection to the HD machine for blood restitution. In a second phase, we explored HD patients' preferences and frontline HD nurses' opinions on the ease of use and safety of both techniques. We hypothesized that there were no differences in risks and preferences between the Y-connection and the usual care technique with regards to CVC infections and other complications.

\section{Materials and methods Study design}

We conducted a prospective, comparative, 3-month point prevalence survey in a 12-bed HD outpatient unit for homedwelling patients, located in a 600-bed teaching hospital in the canton of Valais. The Human Research Ethics Committee of the Canton Valais approved the study protocol in November 2015 (CCVEM 040/15). The study was conducted following the guidelines of Strengthening the Reporting of Observational Studies in Epidemiology. ${ }^{19}$

\section{Population and sample}

A total of 18 eligible patients and 12 frontline nurses in a single HD unit of a Valais hospital were invited to participate. 
Participation was voluntary, and both groups of participants gave their written informed consent.

Patients were eligible if they: 1) were adult men or women with a tunneled CVC for HD, 2) visited the HD unit three times a week or more, 3) had signed a written informed consent form, and 4) understood and spoke French. Frontline nurses were recruited in collaboration with the HD unit's head nurse. HD nurses were eligible and invited to participate in the study if they: 1) had worked in the HD unit for longer than 3 months and 2) were employed more than half-time in the HD unit. All eligible and consenting HD nurses underwent a standardized training program on both techniques and obtained an excellent inter-rater reliability, with a kappa $>0.8 .^{20}$

\section{Allocation to the Y-connection versus usual technique}

After giving their written informed consent, HD patients received sealed envelopes assigning them to the Y-connection or usual technique groups, based on block randomization. Participants were not allowed to change their allocated group. Figure 1 presents the recruitment and allocation process.

\section{Sample size}

Sample size was calculated based on the detection of a difference between the $\mathrm{C}$-reactive protein (CRP) point prevalence values at the baseline assessment and at an assessment after 3 months of using the two techniques. Considering a $20 \%$

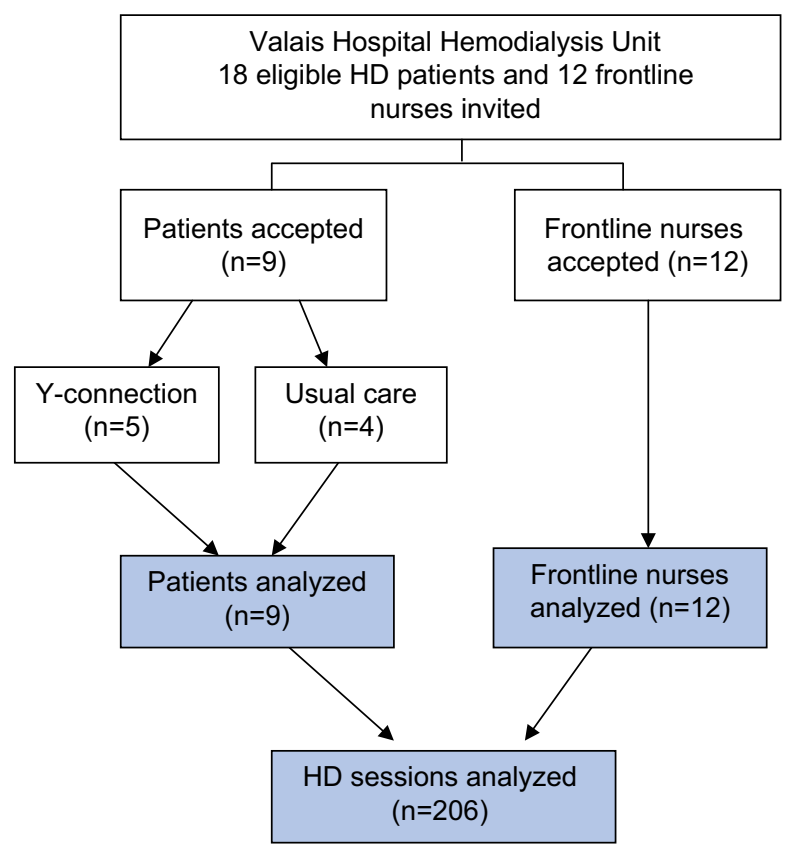

Figure I Flow chart describing the recruitment of the HD patients and frontline nurses.

Abbreviation: HD, hemodialysis. difference in the CRP levels between usual care and the Y-connection blood restitution technique, a sample size of at least $80 \mathrm{HD}$ sessions per technique was required to demonstrate a difference using a two-sided alpha of 0.05 and a power of $80 \%$.

\section{Data collection}

Data were collected from January 1 to March 31, 2016. At the end of the study, frontline nurses completed a questionnaire to assess their preferences and the ease of use of the two techniques. Ease of use was defined as the degree to which a person believed that using a particular system would effortless. $^{21}$ In addition, patients filled in a self-reported questionnaire on their preferences and satisfaction with the techniques applied.

\section{Patient-level data}

We collected patient-level data by asking them questions and looking at their medical records. We recorded sociodemographic data (sex, age), health status (Charlson comorbidity index, primary diagnosis), and the tunneled CVC insertion site. All other data were collected prospectively.

\section{Definitions of complications and adverse outcomes}

CVC was associated with pathological CRP values $(>5 \mathrm{~g} / \mathrm{L})$ with no evidence of alternative sources of infection, similar to the established reporting standards. ${ }^{5,22} \mathrm{CVC}$ infection of the exit site was identified if there was evidence of exudate (with or without pain), crusting, erythema, or induration. CVC tunnel infections were defined by pain, redness, or induration along the subcutaneous course of the line, with or without exudates at the exit site. ${ }^{22-24} \mathrm{CVC}$ dysfunction was identified as consistently suboptimal blood flow of $<250 \mathrm{~mL} / \mathrm{min}$ and/ or declining dialysis performance. ${ }^{18} \mathrm{~A}$ standard X-ray was used to exclude catheter displacement and kinking. ${ }^{5,25,26} \mathrm{We}$ collected data on complications at each HD session and adverse outcomes in relationship to the respective techniques.

\section{Blinding}

Blinding was impractical in this study. Direct clinical observation of the technique applied did not allow blind data collection. However, clinical data from patient records were collected as blindly as possible.

\section{Care procedure and material}

Nurses wore sterile gloves and masks during every application of the two techniques. The frontline HD nurses strictly respected 
the standardized handling protocols for the two blood restitution techniques. The time required to carry out each technique was recorded, as were adverse events. The frontline HD nurses were allowed to note their comments after each HD session.

\section{Usual care technique and Y-connection technique}

The usual care technique (Figure S1) applies a connection technique "open air" between the CVC and the HD machine during the blood restitution and the medication administration and consequently increasing infection risks. The Y-connection technique (Figure S2) is a "closed system" technique between the CVC and the HD machine during the blood restitution and medication administration and consequently decreasing air exposure and infection risk.

\section{Statistical analysis}

Baseline statistics included the study population and their characteristics. Parametric tests were applied to normally distributed variables; in cases of abnormal distributions, nonparametric tests were preferred. Due to the limited number of patients in the HD unit with a CVC, statistical analyses were based on the number of HD sessions attended by the HD patients. To respond to our research questions, we pre- pared descriptive statistics of the number of adverse clinical events occurring during the study period. The relative risks of the two different blood restitution techniques used were computed in a contingency table. Results were considered statistically significant when $p<0.05$. All $p$ values were based on two-tailed tests. The incidence of catheter-related bloodstream infections (CRBSI) in each group was calculated, and the risk ratio (RR) and its $95 \%$ CI were estimated. Computations were performed using SPSS 22.0 software.

\section{Results}

\section{HD patients and frontline nurses}

Nine out of 18 eligible HD patients gave their consent to participate in the study; all 12 eligible frontline nurses agreed to participate.

\section{Baseline sociodemographic and health status data for the HD patients}

The HD patient group was composed of six men and three women, with an average age of 68.3 years old $(\mathrm{SD}=12.3)$. As expected, the majority of the HD patients showed moderate to high scores on the Charlson comorbidity index. Table 1 presents more details of the HD patient group's baseline sociodemographic and health characteristics.

Table I Baseline sociodemographic and health characteristics of HD patients $(n=9)$

\begin{tabular}{|c|c|c|c|}
\hline Variables & $\begin{array}{l}\text { HD patients usual care } \\
\text { technique }\end{array}$ & $\begin{array}{l}\text { HD patients Y-connection } \\
\text { technique }\end{array}$ & $p$-value \\
\hline \multicolumn{4}{|l|}{ Age (years) } \\
\hline Mean (SD) & $61.9(12.5)$ & $72.3(10.9)$ & $<0.00 I^{*, 2}$ \\
\hline Minimum & 43 & 52 & \\
\hline Maximum & 70 & 84 & \\
\hline \multicolumn{4}{|l|}{ Charlson comorbidity index } \\
\hline$\leq 4$ & 3 & 1 & $<0.00 I^{*, a}$ \\
\hline$>5$ & 1 & 4 & \\
\hline \multicolumn{4}{|l|}{ Catheter age (in days) } \\
\hline Mean (SD) & $762(622)$ & $254(173)$ & $0.393^{\mathrm{a}}$ \\
\hline Minimum & 99 & 40 & \\
\hline Maximum & 1335 & 517 & \\
\hline \multicolumn{4}{|l|}{ Health status } \\
\hline Lupus nephritis stage IV & 2 & & \\
\hline Hypertensive nephroangiosclerosis & & 2 & \\
\hline Chronic kidney disease, stage $\mathrm{V}$ & & 1 & NA \\
\hline Diabetic nephropathy and nephrosclerosis & 1 & $\mathrm{I}$ & \\
\hline Proliferative glomerulonephritis & & 1 & \\
\hline Ischemic nephropathy & 1 & - & \\
\hline \multicolumn{4}{|c|}{ Baseline C-reactive protein at study entry } \\
\hline Normal level (<5 mg/L) & 1 & 2 & $0.386^{\mathrm{b}}$ \\
\hline Pathological level ( $\geq 5 \mathrm{mg} / \mathrm{L})$ & 3 & 3 & \\
\hline
\end{tabular}

Notes: ${ }^{M}$ Mann-Whitney U-test. ${ }^{\text {}}$ Chi-square. *Significance $p \leq 0.05$.

Abbreviations: HD, hemodialysis; NA, not applicable. 


\section{The blood restitution technique}

$\mathrm{HD}$ access among the participating patients took place via five jugular, three clavicular, and one trans-lumbar tunneled CVCs. A total of 206 blood restitutions was assessed, involving 73 using the usual technique and 133 using a Y-connection. The hospitalization for a CVCI septicemia of one HD patient in the usual blood restitution group, during the data collection period, explained this imbalance between the two technics $(p \leq 0.001)$.

\section{Assessment of complications}

Table 2 reports on the complications which occurred during the blood restitution activities. At the end of the study, we found no differences between the two techniques with regards to CRP levels ( $p=0.465$ ), local complications due to dressings soiled by exudate at the catheter insert point $(p=0.066)$, or pain $(p=1.00)$. However, HD patients in the usual technique group showed more crusting, erythema, or complications due to induration at the exit site of the tunneled CVC than patients in the Y-connection group $(p=0.006)$. No catheter displacement or kinking of the tunneled CVC occurred during the study.

\section{Associations between the restitution technique, CRB infections, and local complications}

The relative $\mathrm{RR}$ of $\mathrm{CVC}$ with regard to the $\mathrm{HD}$ technique used indicated that CVC was 1.667 times more present among HD patients using the Y-connection than those using the usual technique $(95 \% \mathrm{CI} ; 0.437,6.358, p=0.50)$. However, there was no significant difference between the two techniques. Neither the Y-connection technique (RR 1.63; [95\% CI; $0.554,4.790] ; p=0.32$ ) nor the usual treatment technique (RR 0.58; [95\% CI; 0.277, 1.217]; $p=0.13$ ) were significant relative risk factors for local complications.
Time, perceptions of safety, ease of use, and the preferred techniques of frontline HD nurses

Table 3 reports nurses' assessments of the time necessary for the different techniques, their perceptions about safety and ease of use, and which technique they preferred. The average time spent setting up the usual treatment technique was 10.9 minutes ( $\mathrm{SD}=4.8)$, compared with 9.4 minutes $(\mathrm{SD}=1.8)$ with the Y-connection technique. No significant difference was found between the mean times spent carrying out dialysis and restitution using the two techniques $(p=0.129)$. Almost all of the nurses preferred the Y-connection technique, finding it safer and easier to apply (Table 3 ).

\section{The preferred techniques of HD patients and frontline nurses}

Remembering that the HD patients and nurses involved had previously been trained in both techniques, an autoadministered questionnaire explored their post-study preferences. Fifty-seven percent of the HD patients stated that they felt safer and more comfortable using the Y-connection

Table 3: Time, perceptions, ease of use, and preferences among frontline nurses $(n=12)$

\begin{tabular}{llll}
\hline Complications & $\begin{array}{l}\text { Usual care } \\
\text { technique } \\
(\mathbf{n}=73)\end{array}$ & $\begin{array}{l}\text { Y-connection } \\
\text { technique } \\
(\mathbf{n}=133)\end{array}$ & -value \\
\hline $\begin{array}{l}\text { Perceptions of safety } \\
\text { Favorable }\end{array}$ & $\mathrm{I}$ & $\mathrm{II}$ & $<\left.0.00\right|^{*, a}$ \\
$\begin{array}{l}\text { Technique's ease of use } \\
\text { Favorable }\end{array}$ & 3 & 9 & $<0.00 I^{*, a}$ \\
Preference among nurses & $\mathrm{I}$ & $\mathrm{II}$ & $<0.00 I^{*, a}$
\end{tabular}

Notes: *Significance $p<0.05$; 'Fisher's exact test.

Table 2: Complications during the HD period among the HD patients $(n=9)$

\begin{tabular}{|c|c|c|c|}
\hline Complications & $\begin{array}{l}\text { HD patients usual care } \\
\text { technique }(n=4)\end{array}$ & $\begin{array}{l}\text { HD patients } Y \text {-connection } \\
\text { technique }(n=5)\end{array}$ & $p$-value \\
\hline \multicolumn{4}{|l|}{ C-reactive protein $* *$} \\
\hline Normal level $(<5$ mg/L) & 2 & 2 & $0.500 * *, a$ \\
\hline Pathological level ( $\geq 5 \mathrm{mg} / \mathrm{L})$ & 2 & 3 & \\
\hline \multicolumn{4}{|l|}{ Soiled dressing } \\
\hline Frequency & 0 & 6 & 0.066 \\
\hline \multicolumn{4}{|l|}{ Local pain } \\
\hline Frequency & 0 & 0 & NA \\
\hline \multicolumn{4}{|c|}{ Crusting/erythema/induration } \\
\hline Frequency & 4 & 0 & $0.006^{*, a}$ \\
\hline \multicolumn{4}{|c|}{ Catheter displacement/kinking } \\
\hline Frequency & 0 & 0 & NA \\
\hline
\end{tabular}

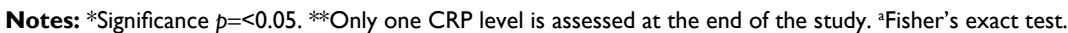
Abbreviations: CRP, C-reactive protein; HD, hemodialysis. 
technique than with their usual HD treatment technique. We hypothesize that the well-known risk of a CRBSI among HD patients probably influenced the majority of participating HD patients to prefer the Y-connection technique. However, the remaining $43 \%$ of the HD patients did not express a preference and trusted that nurses would apply the most appropriate technique. Eleven out of the 12 nurses involved preferred the Y-connection technique and felt that it was safer and easier in use.

\section{Discussion}

To the best of our knowledge, this is the first study comparing the outcomes of complications in relation to two nursing techniques for blood restitution among HD patients fitted with a CV. In addition, this study sought to support EBP by considering nurses' and patients' perceptions and preferences with regard to each technique's ease of use.

The present study's main findings revealed no differences between the usual care and Y-connection techniques with regards to the biological and clinical complications associated with them. The time taken to carry out dialysis and blood restitution using both techniques confirmed our null hypothesis. One of the participants in the usual care group did develop a catheter-related bloodstream septicemia during the study period, however. Nevertheless, this phenomenon should be considered as an intrinsic hazard, in this study, as it is highly prevalent in the CVCs of HD patients, and this is largely documented elsewhere. ${ }^{11,27}$

Extremely rapid innovation is occurring in the field of medical and nursing devices; hospital units are becoming even more high-tech. At the same time, the health care system expects the highest standards of nursing and outcomes in those units, as documented in the study by Saudan et al. ${ }^{1}$ Unfortunately, the literature pays little attention to how effective medical or nursing connection devices are at preventing adverse effects, their ease of use, or their safety for HD patients fitted with a CVC. ${ }^{28} \mathrm{~A}$ recent systematic review by Ravani et al stated that "persons using catheters for HD seem to have the highest risks for death, infections and cardiovascular events compared with other vascular access types" (p. 465). ${ }^{10}$ Although most of the available literature focuses on the types of catheters, it does not consider their ease of use and safety or whether they increase the quality of care and corresponding outcomes. In further research, HD nurses should explore other targeting determinants and risks of complications, such as the ease of use of connections and catheters during HD.
Bearing in mind the nurses' desire to improve EBP, our study considered patients and nurses preferences and perceptions as valuable clinical results. Unfortunately, a triangulation of the EBP components (nurses' preferences, patient preferences, and research results) to create a common statement, result, and conclusion in an issue in our study. Indeed, each component has his own measure and assessment strategy and it seems at this stage not recommendable and feasible to create a composite variable to sustain a general result. Consequently, there is a risk that an economic or cost consideration could be a major determinant in the choice of a standardized connection technique for blood restitution for HD patients. ${ }^{26}$

This study allowed us to highlight the importance of using an EBP approach and the key role that nurses can play in preventing CVCIs among HD outpatients. Furthermore, the study's evaluation and comparison of two HD blood restitution techniques aimed to standardize practice and guide HD nurses seeking to optimize their EBP.

\section{Limitations}

In addition to the relatively small sample size in a small HD unit, the present study was composed of a heterogeneous sample (age and comorbidities) of home-dwelling, older adult, HD outpatients. Home living conditions, nutrition, and daily activities such as changing soiled catheter dressings or personal hygiene between the HD sessions could have influenced the risk of infection. Another limitation was that blood cultures or wound smear examinations were not done at the beginning and end of the study. Finally, any generalization and transfer of the present findings to other HD patients or HD units should be done with caution.

\section{Conclusion}

This study found no differences in the rates of complications between two blood restitution techniques following HD: using a Y-connection or the usual care technique among patients with a CVC. HD patients and nurses expressed their preferences for the Y-connection, and this should be considered when preparing the recommendations for the evidence-based guidelines on blood restitution in HD patients fitted with a CVC.

\section{Acknowledgments}

We thank the patients, the frontline nurses, and the HD unit's nursing supervisor Louise White of the Valais Hospital of Sion for their participation in this study. This study was funded in part by Valais Hospitals. 


\section{Authors' contributions}

HV and NT-J contributed to the study concept, data analysis and interpretation, manuscript drafting, statistical analysis, and fundraising. Both authors contributed to the data acquisition. Both the authors contributed to drafting and revising the manuscript.

\section{Disclosure}

The authors report no conflicts of interest in this work.

\section{References}

1. Saudan P, Halabi G, Perneger T, et al. Variability in quality of care among dialysis units in western Switzerland. Nephrol Dial Transplant. 2005;20(9):1854-1863.

2. Saudan P, Kossovsky M, Halabi G, Martin PY, Perneger TV; Group WSDS. Quality of care and survival of haemodialysed patients in western Switzerland. Nephrol Dial Transplant. 2008;23(6):1975-1981.

3. Golshayan D, Paccaud F, Wauters JP. Epidémiologie de l'insuffisance rénale terminale: comparaison entre deux cantons suisses. Néphrologie. 2002;23(4):179-184. French.

4. Saudan P, Martin PY. Hémodialyse: perspectives futures. Rev Med Suisse. 2008;4:44-48.

5. Vanholder R, Canaud B, Fluck R, et al. Diagnosis, prevention and treatment of haemodialysis catheter-related bloodstream infections (CRBSI): a position statement of European Renal Best Practice (ERBP). NDT Plus. 2010;3(3):234-246.

6. Zingg W, Cartier-Fassler V, Walder B. Central venous catheter-associated infections. Best Pract Res Clin Anaesthesiol. 2008;22(3):407-421.

7. Trautmann M, Moosbauer S, Schmitz FJ, Lepper PM. Experimental study on the safety of a new connecting device. Am J Infecti Control. 2004;32(5):296-300.

8. Eggimann P, Sax H, Pittet D. Catheter-related infections. Microbes Infect. 2004;6(11):1033-1042.

9. Korkor AB, Bretzmann CM. Prevention of hemodialysis catheter-related infection: an unmet need. Dial Transplant. 2009;38(9):377-379.

10. Ravani P, Palmer SC, Oliver MJ, et al. Associations between hemodialysis access type and clinical outcomes: a systematic review. J Am Soc Nephrol. 2013;24(3):465-473.

11. Landry D, Braden G. Reducing Catheter-Related Infections in Hemodialysis Patients. Clin J Am Soc Nephrol. 2014;9(7):1156-1159.
12. Akoh JA. Vascular access infections: epidemiology, diagnosis, and management. Curr Infect Dis Rep. 2011;13(4):324-332.

13. bin SASI, Mohammed JK. An education intervention to improve nurses' knowledge to reduce catheter-related bloodstream infection in hemodialysis unit. Int J Sci Res. 2015;4(4):2263-2282.

14. Jeong IS, Park SM, Lee JM, Song JY, Lee SJ. Effect of central line bundle on central line-associated bloodstream infections in intensive care units. Am J Infectn Control. 2013;41(8):710-716.

15. Sugahara S, Kanno Y, Moriwaki K, et al. Multiple factors affect 3-year survival of patients on chronic hemodialysis. Hemodial Int. 2005;9(1):93-93.

16. Pisoni RL, Zepel L, Port FK, Robinson BM. Trends in US vascular access use, patient preferences, and related practices: an update from the US DOPPS practice monitor with international comparisons. Am J Kidney Dis. 2015;65(6):905-915.

17. Bays CL, Hermann CP. An evidence-based practice primer for infusion nurses. J Infus Nurs. 2010;33(4):220-225.

18. Böhlke M, Uliano G, Barcellos FC. Hemodialysis catheter-related infection: prophylaxis, diagnosis and treatment. JVasc Access. 2015;16(5):347.

19. von Elm E, Altman DG, Egger M, Pocock SJ, Gotzsche PC, Vandenbroucke JP. The Strengthening the Reporting of Observational Studies in Epidemiology (STROBE) statement: guidelines for reporting observational studies. J Clin Epidemiol. 2008;61(4):344-349.

20. Cohen J. Statistical Power and Analysis for the Behavioral Sciences. 2nd ed. Hillsdale, New York, NY: Lawrence ERdbaum Associates; 1988.

21. Davis FD. Perceived usefulness, perceived ease of use, and user acceptance of information technology. MIS Quarterly. 1989;13(3):319-340.

22. Napalkov P, Felici DM, Chu LK, Jacobs JR, Begelman SM. Incidence of catheter-related complications in patients with central venous or hemodialysis catheters: a health care claims database analysis. $B M C$ Cardiovasc Disord. 2013;13(1):1-10.

23. Mermel LA. What is the predominant source of intravascular catheter infections? Clin Infect Dis. 2011;52(2):211-212.

24. Noimark S, Dunnill CW, Wilson M, Parkin IP. The role of surfaces in catheter-associated infections. Chem Soc Rev. 2009;38(12):3435-3448.

25. Verhage AH, van Bommel EF. Catheter fracture - an underrecognized and serious condition in haemodialysis. Nephrol Dial Transplant. 2000;15(6):901-903.

26. Chan MR. Hemodialysis central venous catheter dysfunction. Semin Dial. 2008;21(6):516-521.

27. Xue H, Ix JH, Wang W, et al. Hemodialysis access usage patterns in the incident dialysis year and associated catheter-related complications. $\mathrm{Am}$ J Kidney Dis. 2013;61(1):123-130.

28. Esteve F, Pujol M, Limón E, et al. Bloodstream infection related to catheter connections: a prospective trial of two connection systems. J Hosp Infect. 2007;67(1):30-34. 


\section{Supplementary material}
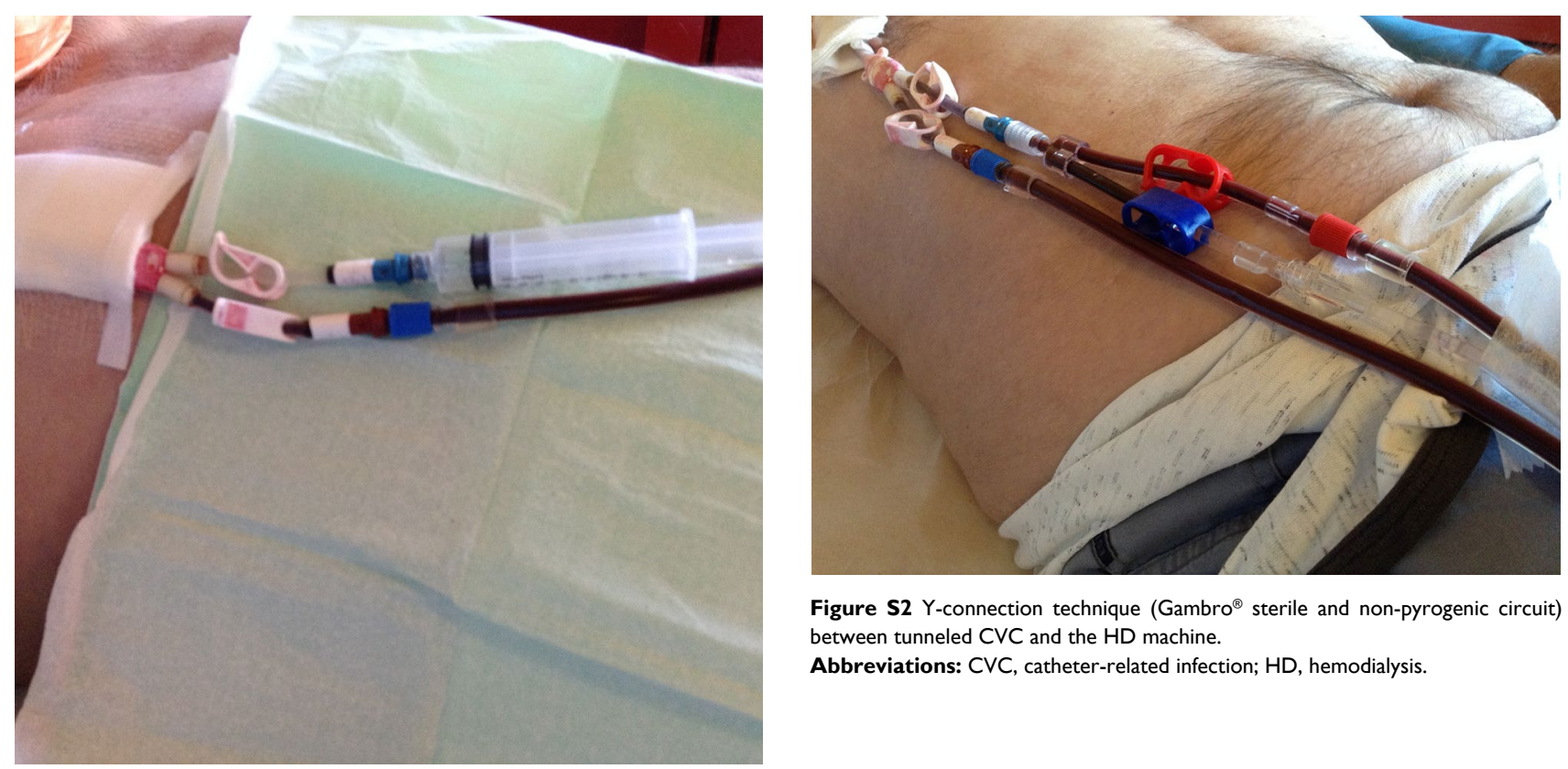

Figure S2 Y-connection technique (Gambro ${ }^{\circledR}$ sterile and non-pyrogenic circuit) between tunneled CVC and the HD machine.

Abbreviations: CVC, catheter-related infection; HD, hemodialysis.

Figure SI Usual care technique with direct access between tunneled CVC and the HD machine.

Abbreviations: CVC, catheter-related infection; HD, hemodialysis.

\section{Publish your work in this journal}

The International Journal of Nephrology and Renovascular Disease is an international, peer-reviewed open access journal focusing on the pathophysiology of the kidney and vascular supply. Epidemiology, screening, diagnosis, and treatment interventions are covered as well as basic science, biochemical and immunological studies. The manuscript management system is completely online and includes a very quick and fair peer-review system, which is all easy to use. Visit http://www dovepress.com/testimonials.php to read real quotes from published authors. 